\title{
(6) OPEN ACCESS \\ Differentiating primary and extragenital metastatic mucinous ovarian tumours: an algorithm combining PAX8 with tumour size and laterality
}

\author{
Ajin Hu, ${ }^{1}$ Hongwei Li, ${ }^{2}$ Letian Zhang, ${ }^{1}$ Caixia Ren, ${ }^{3}$ Yuxiang Wang, ${ }^{1}$ Yan Liu, \\ Congrong Liu ${ }^{1}$
}

${ }^{1}$ Department of Pathology, School of Basic Medical Science, Peking University Third Hospital, Peking University Health Science Centre, Beijing, China

2Department of Pathology, Beijing Chuiyangliu Hospital, Beijing, China

${ }^{3}$ Department of Histology and Embryology, Peking University Health Science Centre, Beijing, China

Correspondence to Dr C Liu, Department of Pathology, Peking University Health Science Centre, 38 College Road, Haidian, Beijing 100191, China; congrong_liu@hsc.pku.edu.cn

Received 12 February 2015 Revised 5 March 2015 Accepted 9 March 2015 Published Online First 31 March 2015

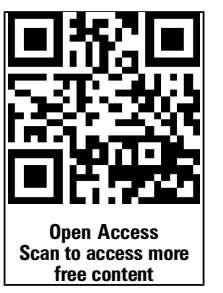

CrossMark

To cite: Hu A, Li H,

Zhang L, et al. I Clin Pathol 2015;68:522-528.

\section{ABSTRACT}

Aims Our aim was to analyse the utility of the algorithm combining PAX8 with clinicopathological characteristics (tumour size, laterality and patient age) in differentiating primary ovarian mucinous tumours (POMTs) from extragenital metastatic mucinous carcinomas involving the ovary (eMOMCs).

Methods and results Immunohistochemical staining for PAX8 was performed on formalin fixed, paraffin embedded tissues from 47 POMTs, 18 eMOMCs and 70 extragenital primary mucinous carcinomas (ePMCs) using anti-PAX8 rabbit polyclonal antibody (pAb) and antiPAX8 rabbit monoclonal antibody (mAb). PAX8 (pAb) positive signals were found in 3/18 eMOMCs and in 32/ 70 ePMCs. PAX8 (mAb) demonstrated superior specificity, with $0 \%$ positivity in both eMOMCs and ePMCs, but unfavourable sensitivity, with $60.9 \%$ in ovarian mucinous borderline tumours and $45.8 \%$ in POMCs. Although PAX8 (mAb) immunostaining status $(66.2 \%)$, tumour size $(75.4 \%)$ and laterality (84.6\%) demonstrated unsatisfactory accuracy when they were evaluated individually in differentiating POMTs from eMOMCs, a combination of PAX8 (mAb) immunostaining status, tumour size and laterality markedly increased accuracy (86.2\%), with a satisfactory Youden Index (63.7\%).

Conclusions PAX8 (mAb) was a specific marker in differentiating POMTs from eMOMCs. As a simple, convenient and high performance to price ratio algorithm, a combination of PAX8 (mAb) immunostaining with tumour size and laterality will improve the diagnostic criteria of ovarian mucinous metastasis.

\section{INTRODUCTION}

The distinction between primary ovarian mucinous tumours (POMTs) and metastatic mucinous carcinomas involving the ovary (MOMCs) is often problematic. ${ }^{1}$ MOMCs commonly metastasise from the alimentary system, breast and cervix, which sometimes produces histological patterns as well as immunohistochemical features closely resembling well differentiated primary ovarian mucinous carcinomas (POMCs), or benign or borderline POMTs. ${ }^{2-6}$ Occasionally, the primary tumours of MOMCs may be clinically silent, only presenting with symptoms related to an ovarian mass, and may not manifest until a period of time after total abdominal hysterectomy with bilateral salpingo-oophorectomy. As a result, it is difficult to differentiate between primary and metastatic mucinous neoplasms based on clinical and histopathology features alone, even though the differential diagnosis is decisive for treatment and prognosis. $^{7}$

Seidman $e \mathrm{al}^{3}$ first recommended a simple algorithm (bilateral tumours of any size, or unilateral tumour $<10 \mathrm{~cm}=$ metastatic; unilateral tumour $\geq 10 \mathrm{~cm}=$ primary) and claimed it could accurately classify $90 \%$ of mucinous neoplasms involving the ovary. However, some cases violate the algorithm. ${ }^{8}$ To deal with an equivocal tumour, immunohistochemistry (IHC) may be helpful, although it is not definitive. ${ }^{9}$ Several candidate markers have been advocated, including PAX8, which is a member of the paired box gene family of transcription factors and plays a critical role in the organogenesis of the Müllerian system. ${ }^{10}$ In addition, PAX8 has the potential to induce tumorigenesis and is expressed in a tissue specific manner during neoplastic transformation. ${ }^{11}{ }^{12}$ Recently, PAX8 has been shown to be constantly expressed in the majority of histological subtypes of Müllerian epithelial tumours. ${ }^{13}$ Absence of PAX8 expression in mammary carcinoma and malignant mesothelioma is especially valuable, considering that ovarian involvement of these tumours is not uncommon. ${ }^{14}{ }^{15}$ Nevertheless, the percentage of PAX8 positivity in POMCs has ranged from $0 \%$ to $50 \%$ in previous studies. ${ }^{14} 16-23$

With the growing awareness that true POMCs appear to be substantially less common than previously reported, we believe that PAX8 expression in POMTs should be explored based on full compliance with stringent morphological criteria, in conjunction with detailed clinical information and close follow-up. The other reason behind the disparity may be the variety of anti-PAX8 antibodies used in different studies. The majority of the published studies used anti-PAX8 rabbit polyclonal antibody (pAb). ${ }^{14}$ 16-22 Unfortunately, currently available commercial pAbs have cross reactions with lymphocytes, metastatic pancreatic cancers, duodenal neuroendocrine tumours and a subset of rectal, gastric and appendiceal neuroendocrine tumours, which could confuse the interpretation and thus influence the confidence and accuracy of the diagnosis. ${ }^{13} 24$

In addition, information about PAX8 expression in different POMTs categories is limited and, to date, no study has been performed to verify PAX8 expression in POMTs, in extragenital metastatic mucinous carcinomas involving the ovary (eMOMCs) or in extragenital primary mucinous carcinomas (ePMCs).

Therefore, our aim was to confirm the exact role of PAX8 in the differential diagnosis of POMTs, 
and to develop an algorithm to improve the accuracy of this clinical practice.

\section{MATERIALS AND METHODS}

\section{Tissue specimens}

Formalin fixed, paraffin embedded tissues were selected from the Department of Pathology, Peking University Health Science Centre. Forty-seven POMTs (23 borderline, 24 carcinomas), 18 eMOMCs ( 7 from the colorectum, 6 from the appendix, 4 from the stomach and 1 from the pancreas) and 70 ePMCs $(29$ colorectal, 16 mammary, 13 gastric, 7 pancreatic, 3 appendiceal and 2 biliary tract) were from patients who underwent surgery from 1999 to 2011.

Twenty-one cases of POMCs were stage I while only two cases were stage II and one was stage IV. All pathological diagnoses for specimens used in this study were reviewed using established histological criteria, and were confirmed by two pathologists. ${ }^{1} 22526$ Briefly, tumours were diagnosed as mucinous borderline tumours (MBTs) when they showed non-invasive tumours with intraglandular or intracystic epithelial proliferation in the absence of disturbing architectural features of the lining epithelium, and with mild to moderate nuclear atypia. A tumour was classified as MBT with intraepithelial carcinoma if a borderline tumour contained foci in which the epithelial lining cells had marked atypical nuclei or had appreciable architectural proliferation but a cribriform pattern on its own or epithelial stratification of greater than three cell layers in the absence of severe atypia. MBTs with microinvasion were diagnosed when a borderline tumour had stromal invasion of $<5 \mathrm{~mm}$ in a contiguous area. Tumours were classified as POMCs when they exhibited stromal invasion of $>5 \mathrm{~mm}$, characterised by an expansile growth pattern or cribriform pattern, without having discrete nodularity. Tumours were classified as MOMCs when they exhibited surface involvement, stromal invasion characterised by a multinodular growth pattern in which the mucinous gland aggregates were separated from each other by regions of preserved ovarian stroma or areas simulating a benign/borderline appearance but displaying significant cytological atypia.

All patients, except those who presented with ePMCs without ovary involvement, were closely followed-up for at least 3 years. All available clinical data and follow-up outcomes were added to determine the primary sites for the metastatic cases and to exclude the possibility of metastatic tumours in diagnosing POMTs. Four cases with equivocal clinicopathological data and an undetermined final diagnosis were excluded. Tumour size (if bilateral involvement, we recorded the size of the larger tumour), laterality and patient age were recorded for all cases of POMTs and eMOMCs.

\section{Immunohistochemistry}

Representative haematoxylin-eosin stained slides from the tumours were reviewed, and one representative block was selected for IHC. Immunohistochemical analyses were performed on formalin fixed, paraffin embedded, $4 \mu \mathrm{m}$ thick tissue sections. The primary antibodies used were anti-PAX8 rabbit monoclonal antibody (mAb) (Zeta, Corporation, California, USA) and pAb (Protein Tech Group, Chicago, Illinois, USA). Tissue sections were deparaffinised and rehydrated through xylenes and graded ethanol solutions to water. Antigen retrieval was performed using pressure cooker pretreatment in Tris EDTA buffer ( $\mathrm{pH} 9.0$ ) for 2 min. After blocking the endogenous peroxidase activity with $3 \%$ hydrogen peroxidase for

Table 1 PAX8 expression in POMTs, eMOMCs and ePMCs

\begin{tabular}{|c|c|c|c|c|c|c|c|c|c|c|c|c|}
\hline & \multicolumn{6}{|l|}{ PAX8 mAb } & \multicolumn{6}{|l|}{ PAX8 pAb } \\
\hline & \multirow[b]{2}{*}{ Positive/total cases (\%) } & \multicolumn{4}{|c|}{ Staining intensity } & \multirow[b]{2}{*}{ p Value } & \multirow[b]{2}{*}{ Positive/total cases ( $\%)$} & \multicolumn{4}{|c|}{ Staining intensity } & \multirow[b]{2}{*}{ p Value } \\
\hline & & 0 & $1+$ & $2+$ & $3+$ & & & 0 & $1+$ & $2+$ & $3+$ & \\
\hline POMTs & $25 / 47(53.2)$ & 22 & 12 & 9 & 4 & $0.3852^{*}$ & $37 / 47(78.7)$ & 10 & 18 & 9 & 10 & $0.7238^{*}$ \\
\hline MBTs & $14 / 23(60.9)$ & 9 & 6 & 5 & 3 & & 19/23 (82.6) & 4 & 8 & 6 & 5 & \\
\hline POMCs & $11 / 24(45.8)$ & 13 & 6 & 4 & 1 & & $18 / 24(75.0)$ & 6 & 10 & 3 & 5 & \\
\hline FIGO stage & & & & & & $1 \dagger$ & & & & & & $<0.5392 \dagger$ \\
\hline la-lc & $10 / 21(47.6)$ & 11 & 5 & 4 & 1 & & $14 / 21(66.7)$ & 7 & 8 & 3 & 3 & \\
\hline II-IV & $1 / 3(33.3)$ & 2 & 1 & 0 & 0 & & $3 / 3(1.0)$ & 0 & 1 & 0 & 2 & \\
\hline eMOMCs & $0 / 18(0)$ & 0 & 0 & 0 & 0 & $<0.001 \ddagger$ & $3 / 18(16.7)$ & 15 & 2 & 1 & 0 & $<0.001 \ddagger$ \\
\hline Colorectal & $0 / 7(0)$ & 0 & 0 & 0 & 0 & & $2 / 7(28.6)$ & 5 & 1 & 1 & 0 & \\
\hline Appendiceal & $0 / 6(0)$ & 0 & 0 & 0 & 0 & & $0 / 6(0)$ & 6 & 0 & 0 & 0 & \\
\hline Gastric & $0 / 4(0)$ & 0 & 0 & 0 & 0 & & $1 / 4(25.0)$ & 3 & 1 & 0 & 0 & \\
\hline Pancreatic & $0 / 1(0)$ & 0 & 0 & 0 & 0 & & $0 / 1(0)$ & 1 & 0 & 0 & 0 & \\
\hline ePMCs & $0 / 70(0)$ & 0 & 0 & 0 & 0 & $<0.0019$ & $32 / 70(45.7)$ & 38 & 10 & 17 & 5 & $<0.019$ \\
\hline Colorectal & $0 / 29(0)$ & 0 & 0 & 0 & 0 & & $12 / 29(41.4)$ & 17 & 4 & 6 & 2 & \\
\hline Gastric & $0 / 13(0)$ & 0 & 0 & 0 & 0 & & $3 / 13(23.1)$ & 10 & 1 & 1 & 1 & \\
\hline Appendiceal & $0 / 3(0)$ & 0 & 0 & 0 & 0 & & $2 / 3(66.7)$ & 1 & 1 & 1 & 0 & \\
\hline Pancreatic & $0 / 7(0)$ & 0 & 0 & 0 & 0 & & $3 / 7$ (42.9) & 4 & 1 & 2 & 0 & \\
\hline Biliary tract & $0 / 2(0)$ & 0 & 0 & 0 & 0 & & $1 / 2(50)$ & 1 & 1 & 0 & 0 & \\
\hline Mammary & $0 / 16(0)$ & 0 & 0 & 0 & 0 & & $11 / 16(68.8)$ & 5 & 2 & 7 & 2 & \\
\hline $\begin{array}{l}\text { Staining intensi } \\
{ }^{*} \text { POMTs (MBTs } \\
\text { †POMCs (FIGO } \\
\text { †POMTs vs eM } \\
\text { §POMTs vs ePI } \\
\text { eMOMCs, extra } \\
\text { borderline tums }\end{array}$ & $\begin{array}{l}\text { s graded 0-3. Nuclear staining } \\
\text { carcinoma), Fisher's exact test } \\
\text { ge la-Ic vs FIGO stage II-IV), } \\
\text { Cs, Fisher's exact test. } \\
\text {, Fisher's exact test. } \\
\text { ital metastatic mucinous carci } \\
\text {; POMCs, primary ovarian mu }\end{array}$ & her's $>5 \%$ & malig & vary; & as cor & genital prin & 更 & & : & & & nous \\
\hline
\end{tabular}


$10 \mathrm{~min}$, the sections were incubated with primary antibody (mAb, dilution: 1:100; pAb, dilution: $1: 100$ ) overnight at $4^{\circ} \mathrm{C}$. Detection reactions utilised the Envision kit from Dako (Dako Cytomation, Glostrup, Denmark). Diaminobenzidine was used as the chromogen and haematoxylin as the counterstain. Appropriate positive (serous ovarian carcinoma tissue) and negative (incubation with Tris buffered saline instead of the specific primary antibody) controls were run simultaneously.

Analyses of IHC results were performed by two independent authors (AH and HL). Discrepancies in analyses were reconciled following a review by a third reviewer (CL). Nuclear staining in more than $5 \%$ of malignant cells was considered positive. For positive cases, staining intensity was further scored as follows: 0 , no staining; 1 , unequivocal but weak intensity; 2 , moderate intensity; and 3, marked intensity.

\section{Statistical methods}

The Kruskal-Wallis test, Mann-Whitney test and Fisher's exact test were used to compare differences in PAX8 expression, tumour size, laterality and patient age in the mucinous tumours. The receiver operator characteristic (ROC) curve was used to detect the sensitivity and specificity of PAX8 (mAb) IHC status, tumour size, laterality and patient age in differentiating POMTs from eMOMCs. The value of the area under the ROC curve (AUC) was between 0 and 1. A test with an AUC value of 0.5-0.7 has weak overall diagnostic performance, an AUC of
0.7-0.9 has moderate overall diagnostic performance and an AUC of 0.9-1 has a favourable overall diagnostic performance. $^{27} \mathrm{~A} p$ value $<0.05$ was considered statistically significant.

\section{RESULTS}

The results of IHC are summarised in table 1. PAX8 staining signals were localised on nuclei of tumour cells (figure 1). PAX8 $(\mathrm{mAb})$ did not show any reactivity with eMOMCs $(0 / 18)$ or ePMCs $(0 / 70)$ tumour cells, while positive signals were seen in $60.9 \%(14 / 23)$ of MBTs and in 45.8\% (11/24) of POMCs. In contrast, PAX8 (pAb) not only stained a high percentage of POMTs $(82.6 \%(19 / 23)$ MBTs and 75\% (18/24) POMCs), but also showed positive signals in $16.7 \%$ (3/18) of eMOMCs and in a high proportion of ePMCs, including 41.4\% (12/29) colorectal, 68.8\% (11/16) mammary, 23.1\% (3/13) gastric, 42.9\% (3/7) pancreatic, 66.7\% (2/3) appendiceal and 50\% (1/2) biliary tract. Additionally, unlike PAX8 pAb, PAX8 mAb did not exhibit any cross reaction with $\mathrm{B}$ cells or pancreatic cells. Although both antibodies demonstrated meaningful roles in the differential diagnosis of mucinous tumours involving the ovary (table 1 ), our data strongly suggested that PAX8 (mAb) was far more specific than PAX8 (pAb), especially in differentiating POMTs from eMOMCs. Consequently, only PAX8 (mAb) IHC results were analysed in the following studies.

Compared with POMTs, eMOMCs were more frequently bilateral $(55.6 \%$ vs $4.2 \%, \mathrm{p}<0.01)$, showed a much smaller
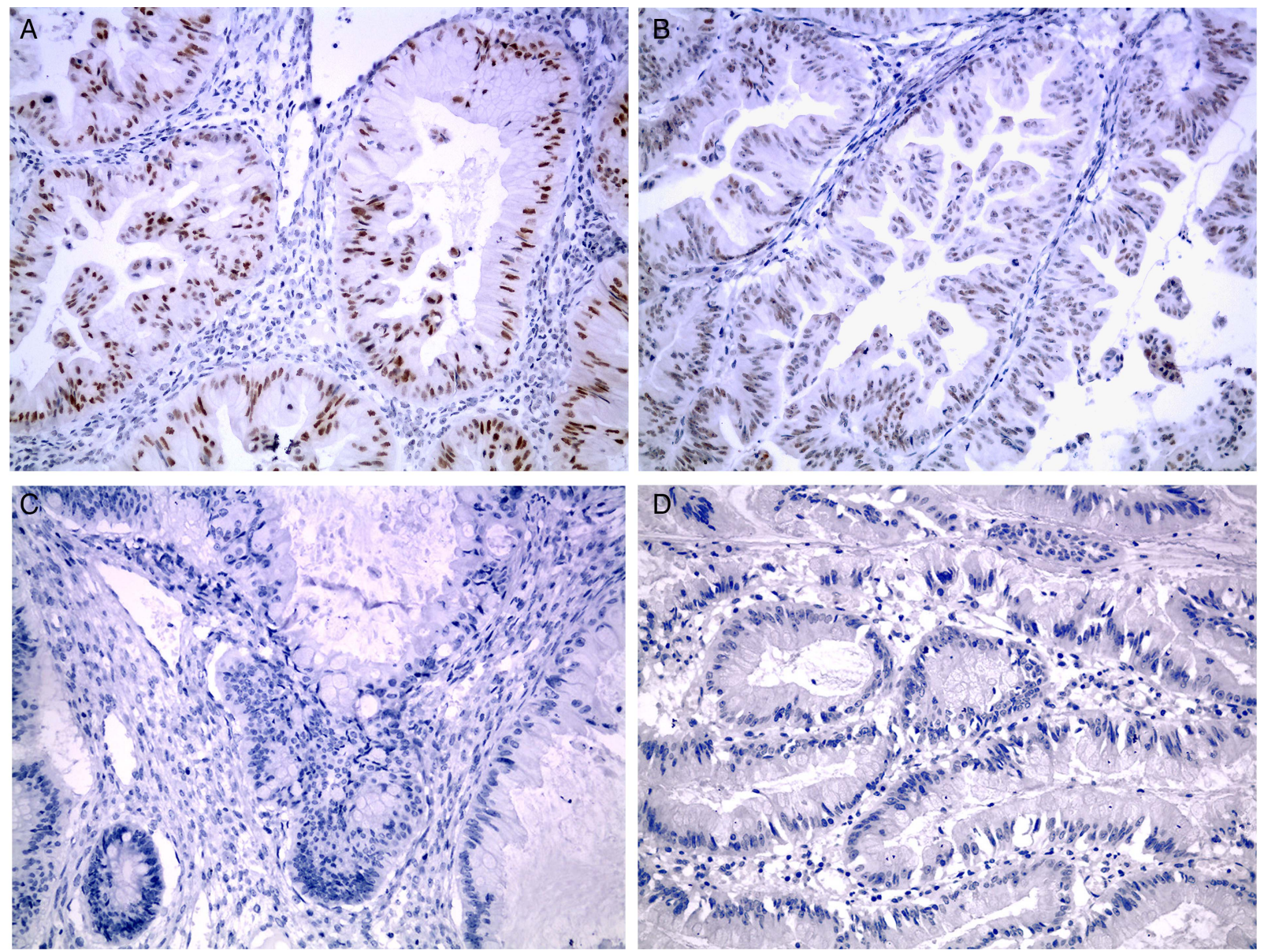

Figure 1 PAX8 (mAb) immunohistochemistry in POMTs, eMOMCs and ePMCs. (A) MBTs: marked PAX8 nuclear reaction. (B) POMCs: moderate PAX8 nuclear reaction. (C) Colorectal MOMCs: negative PAX8 reaction. (D) Colorectal PMCs: negative PAX8 reaction. eMOMCs, extragenital metastatic mucinous carcinomas involving the ovary; ePMCs, extragenital primary mucinous carcinomas; mAb, monoclonal antibody; MBTs, ovarian mucinous borderline tumours; MOMCs, metastatic mucinous carcinomas involving the ovary; PMCs, primary mucinous carcinomas; POMCs, primary ovarian mucinous carcinomas; POMTs, primary ovarian mucinous tumours. 

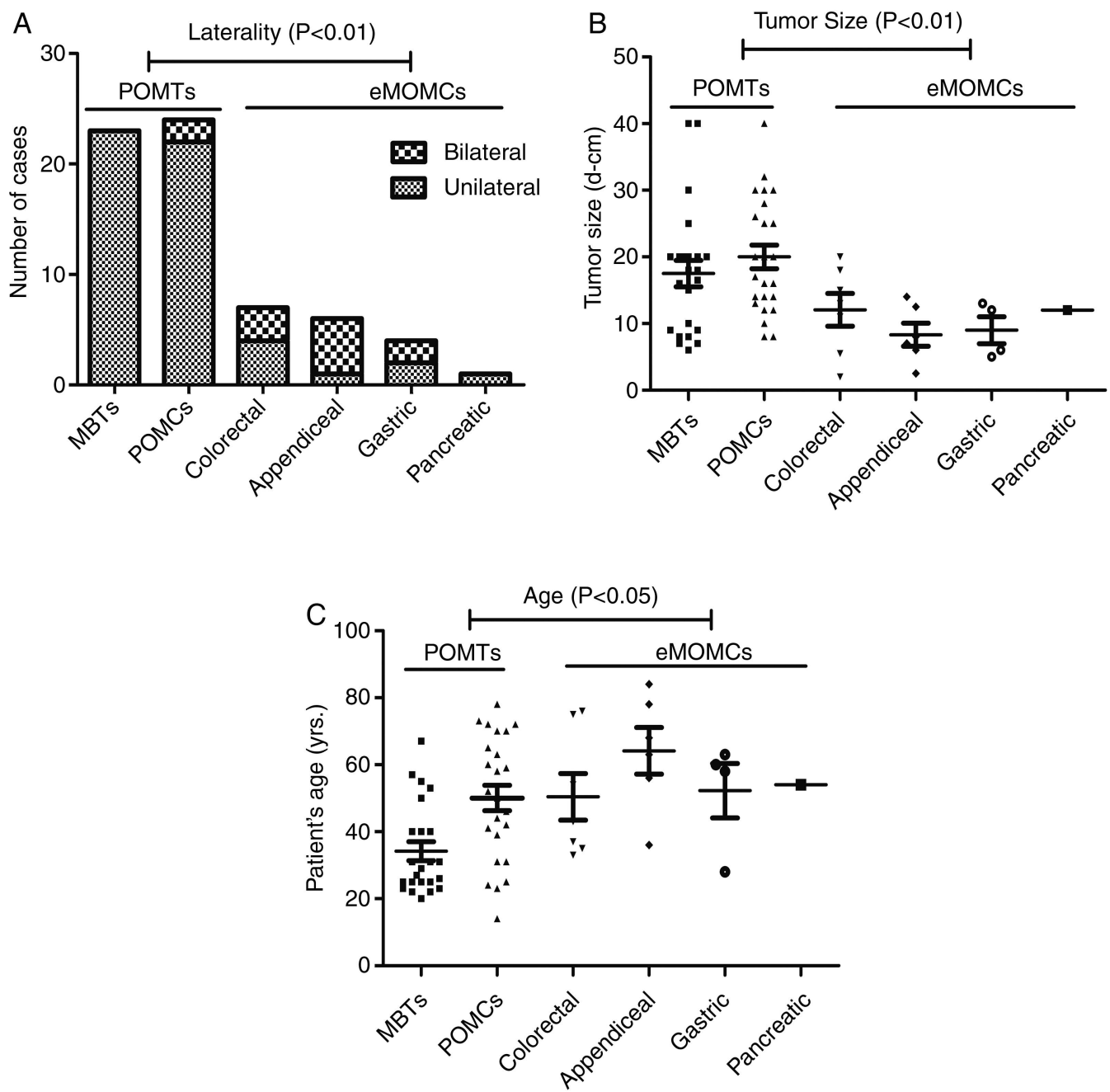

Figure 2 Tumour laterality, size and patient age in POMTs and eMOMCs. (A) eMOMCs more frequently showed bilateral involvement compared with POMTs $(p<0.01)$. (B) Tumour sizes of eMOMCs were much smaller than those of POMTs $(p<0.01)$. (C) Patient age was higher for eMOMCs compared with POMTs $(p<0.05)$. eMOMCs, extragenital metastatic mucinous carcinomas involving the ovary; MBTs, ovarian mucinous borderline tumours; POMCs, primary ovarian mucinous carcinomas; POMTs, primary ovarian mucinous tumours.

mean tumour size $(11.1$ vs $18.8 \mathrm{~cm}, \mathrm{p}<0.01)$ and presented at a higher mean age $(51.8$ vs 40.6 years, $\mathrm{p}<0.05)$ (figure 2$)$. Based on AUC, PAX8 (mAb) IHC status $(0.766,95 \%$ CI 0.654 to $0.878 ; \mathrm{p}<0.01)$, tumour size $(0.870,95 \%$ CI 0.779 to 0.961 ; $\mathrm{p}<0.01)$, laterality $(0.757,95 \%$ CI 0.605 to $0.908 ; \mathrm{p}<0.01)$ and patient age $(0.645,95 \%$ CI 0.497 to $0.792 ; \mathrm{p}=0.073)$ were assessed (figure 3). All of the above parameters, except patient age (AUC <0.7), demonstrated some success in distinguishing POMTs from eMOMCs. However, the accuracy of each individual parameter $(66.2 \%$ for PAX8 (mAb) IHC status, $75.4 \%$ for tumour size and $84.6 \%$ for laterality) and the accuracy of the algorithm proposed by Seidman et $a l^{3}$ (76.6\% for POMTs and $72.2 \%$ for eMOMCs) were both unsatisfactory in our cohort. We then tried combinations of the above parameters. Interestingly, when PAX8 (mAb) IHC status was combined with tumour size and laterality, diagnostic accuracy increased markedly to $86.2 \%$, with the highest Youden Index (63.7\%), which was superior to all other combinations (table 2).

\section{DISCUSSION}

Our study confirmed the most frequent primary sources of eMOMCs as colorectum $(38.9 \%, 7 / 18)$, followed by appendix
$(33.3 \%, 6 / 18)$ and upper gastrointestinal tract $(27.8 \%, 5 / 18)$. MOMCs from these organs can be potentially difficult to diagnose, as their primary tumours may be insidious and clinically silent, and they are often diagnosed synchronously with or even after the ovarian tumours. ${ }^{1}$ In our cohort, 6 of 18 cases of eMOMCs were misdiagnosed as POMCs at presentation and were proved to be metastatic lesions 1-3 years later. MOMCs are always associated with increased aggressiveness, and their behaviour is diametrically opposite to that of the majority of POMCs, which are in most cases at an early stage of diagnosis. ${ }^{28}$

Most $(95.8 \%, 23 / 24)$ of our POMCs were FIGO stages I and II at presentation. Consistent with previous studies, POMCs appear to develop from benign and borderline POMTs, which tend to be the largest ovarian masses overall and usually involve only one ovary; MOMCs are often much smaller and bilateral. As a result, it would be of great value to distinguish POMTs from MOMCs in the intraoperative diagnosis. However, difficulty distinguishing POMTs from MOMCs can extend beyond the intraoperative period, even to when permanent sections are evaluated. Some MOMCs, especially from pancreatic and intestinal primary tumours, can closely mimic POMTs morphologically and can occur in the absence of a known extra-ovarian 
A ROC Curve of PAX8 (mAb), Laterality and Patient's Age
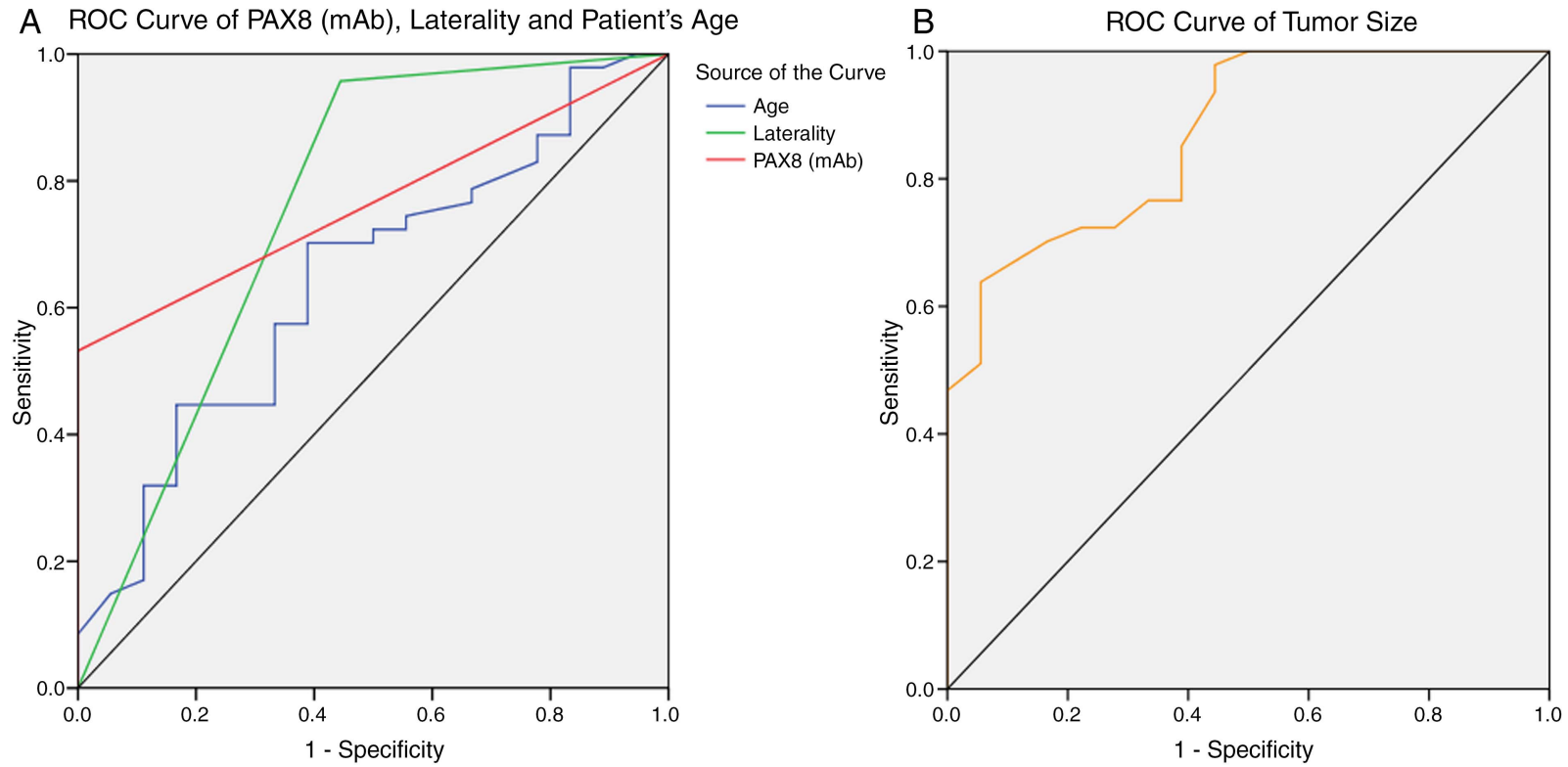

Figure 3 Receiver operator characteristic (ROC) curve analysis of PAX8 (mAb) immunohistochemistry status (positive /negative), tumour size, laterality and patient age for distinguishing POMTs from eMOMCs. The chance diagonal (the line segment from 0,0 to 1,1$)$ has an area under the ROC curve (AUC) of 0.5 . (A) AUCs of PAX8 (mAb), laterality and patient age were $0.766(95 \% \mathrm{Cl} 0.654$ to $0.878 ; \mathrm{p}<0.001), 0.757(95 \% \mathrm{Cl} 0.605$ to $0.908 ; p<0.001)$ and $0.645(95 \% \mathrm{Cl} 0.497$ to $0.792 ; p=0.021)$, respectively. (B) AUC of tumour size was $0.870(95 \% \mathrm{Cl} 0.779$ to $0.961 ; p<0.001)$. eMOMCs, extragenital metastatic mucinous carcinomas involving the ovary; mAb, monoclonal antibody; POMTs, primary ovarian mucinous tumours.

primary site. ${ }^{4-6}$ Thus, to correctly and properly diagnose problematic cases, it is essential to use more advanced molecular markers in clinical practice.

PAX8 has been shown to be a sensitive and specific differential diagnostic marker for carcinomas of ovarian origin. ${ }^{14} 151929$ Chu et $a l^{20}$ proved that PAX8 expression was only found in gynaecology mucinous carcinomas but not in other types of mucinous carcinomas. However, the amount of PAX8 positivity (ie, $<50 \%$ ) demonstrated great discrepancy due to various morphological criteria and the multifarious anti-PAX8 antibodies used in different studies of POMTs (table 3). The diagnoses of POMTs and eMOMCs of our cohort were rigorously based on stringent histological criteria and were validated by detailed clinical records as well as long term ( $\geq 3$ years) follow-up.

Compared with previously published data, our study demonstrated unusually high expression of PAX8 (pAb) in POMTs and should be further validated in more studies. ${ }^{14} 22$ Also, pAb demonstrated unsatisfactory specificity in our study and was eliminated from our final analysis. Consistent with previous studies, less than half $(45.8 \%, 11 / 24)$ of our POMCs reacted with PAX8 (mAb). The lower positivity of PAX8 in POMCs compared with ovarian serous (93-100\%), endometrioid (83-100\%) and clear cell (100\%) carcinomas supports the theory that POMCs are unique among other epithelial ovarian carcinomas subtypes. ${ }^{14-18}$ Moreover, PAX8 is a lineage restricted transcription factor that plays an essential role in the organogenesis of the Müllerian system. ${ }^{10} 12$ In the reproductive tract, PAX8 expression was shown to be restricted to secretory cells of the fallopian tube epithelium which has recently been suggested to be cell origins for most serous ovarian carcinomas. ${ }^{19} 30$ Recent studies have provided evidence that microscopic transitional cell nests at the tubal-peritoneal junction or fimbrial epithelial cells of the fallopian tube could be the potential source of POMCs. ${ }^{31} 32$ This would be consistent with their non-Müllerian appearance and

Table 2 Combined analysis of the validity of PAX8 (mAb), tumour size and laterality in distinguishing POMTs from eMOMCs

\begin{tabular}{|c|c|c|c|c|c|c|c|c|}
\hline & $\begin{array}{l}\text { POMTs } \\
\text { (n) }\end{array}$ & $\begin{array}{l}\text { MOMCs } \\
\text { (n) }\end{array}$ & $\begin{array}{l}\text { Sensitivity } \\
(\%)\end{array}$ & $\begin{array}{l}\text { Specificity } \\
(\%)\end{array}$ & $\begin{array}{l}\text { PPV } \\
(\%)\end{array}$ & $\begin{array}{l}\text { NPV } \\
(\%)\end{array}$ & $\begin{array}{l}\text { Accuracy } \\
(\%)\end{array}$ & $\begin{array}{l}\text { Youden } \\
\text { Index (\%) }\end{array}$ \\
\hline Unilateral=primary, bilateral=metastatic & 45 & 10 & 95.7 & 55.6 & 84.9 & 83.3 & 84.6 & 51.3 \\
\hline$\geq 10 \mathrm{~cm}=$ primary,$<10 \mathrm{~cm}=$ metastatic & 38 & 11 & 80.9 & 61.1 & 84.4 & 55.0 & 75.4 & 42 \\
\hline PAX8 mAb (+)=primary, PAX8 mAb (-)=metastatic & 25 & 18 & 54.2 & 100.0 & 100.0 & 45.0 & 66.2 & 54.2 \\
\hline $\begin{array}{l}\text { Unilateral } \geq 10 \mathrm{~cm}=\text { primary, bilateral tumours of any size or } \\
\text { unilateral tumour }<10 \mathrm{~cm}=\text { =metastatic }\end{array}$ & 36 & 13 & 76.6 & 72.2 & 87.8 & 51.2 & 75.4 & 48.8 \\
\hline $\begin{array}{l}\text { PAX8 mAb (+) or PAX8 mAb (-) and } \geq 10 \mathrm{~cm}=\text { primary, PAX8 mAb } \\
(-) \text { and }<10 \mathrm{~cm}=\text { metastatic }\end{array}$ & 43 & 11 & 91.5 & 61.1 & 86 & 73.3 & 83.1 & 52.6 \\
\hline $\begin{array}{l}\text { PAX8 mAb (+) orPAX8 mAb }(-) \text { and unilateral=primary, PAX8 } \\
\text { mAb (-) and bilateral=metastatic }\end{array}$ & 45 & 10 & 95.7 & 55.5 & 84.9 & 83.3 & 84.6 & 51.2 \\
\hline $\begin{array}{l}\text { PAX8 mAb (+) or PAX8 mAb (-) and unilateral } \geq 10 \mathrm{~cm}=\text { primary, } \\
\text { PAX8 mAb (-) and bilateral tumours of any size or PAX8 mAb }(-) \\
\text { and unilateral tumour }<10 \mathrm{~cm}=\text { metastatic }\end{array}$ & 43 & 13 & 91.5 & 72.2 & 89.6 & 87 & 86.2 & 63.7 \\
\hline
\end{tabular}


Table 3 Previously published results of PAX8 expression in ovary mucinous tumours (positive/total cases)

\begin{tabular}{|c|c|c|c|c|}
\hline Previous studies & Antibodies & $\begin{array}{l}\text { Ovary benign mucinous } \\
\text { tumours }\end{array}$ & $\begin{array}{l}\text { Ovary benign/borderline } \\
\text { mucinous tumours }\end{array}$ & $\begin{array}{l}\text { Ovary mucinous } \\
\text { carcinomas }\end{array}$ \\
\hline Bowen et $a l^{19}$ & $\alpha$-mPax8-bllI,pAb* & ND & ND & $2 / 18$ \\
\hline Nonaka et al ${ }^{14}$ & RpAbt & ND & ND & $1 / 12$ \\
\hline Köbel et $a l^{22}$ & $\alpha$-mPax8-bIII,pAb* & ND & ND & $2 / 31$ \\
\hline Tabrizi et $a l^{21}$ & $\mathrm{pAb} *$ & ND & ND & $0 / 30$ \\
\hline Laury et $a l^{15}$ & $\mathrm{RpAbt}$ & ND & ND & $10 / 25$ \\
\hline Tong et $a l^{29}$ & RpAbt & ND & ND & $0 / 2$ \\
\hline Tacha et $a l^{18}$ & $\mathrm{RpAb} \ddagger$ & $0 / 1$ & ND & $27 / 54$ \\
\hline Ozcan et $a l^{13}$ & RpAbt & ND & $6 / 31$ & $2 / 9$ \\
\hline Chu et $a l^{20}$ & RpAb§ & ND & ND & 9/19 \\
\hline Liliac et $a l^{23}$ & $\mathrm{RpAbt}$ & ND & ND & $4 / 13$ \\
\hline Total (\%) & & $0 / 1(0)$ & 6/31 (19.4) & $57 / 213(26.76)$ \\
\hline
\end{tabular}

low PAX8 expression compared with ovarian non-mucinous carcinomas. Moreover, PAX8 (mAb) should be considered as having limited reliability in distinguishing POMTs from eMOMCs due to its good specificity but poor sensitivity.

Unfortunately, in our cohort, the algorithm proposed by Seidman et $a l^{3}$ correctly classified only $75.4 \%$ of mucinous tumours involving the ovary $(76.6 \%$ of POMTs and $72.2 \%$ of eMOMCs). Our ROC curve analysis supported the fact that PAX8 (mAb) IHC status, tumour size and laterality were useful in differentiating POMTs and eMOMCs, even though their individual accuracy was disappointing. The combination of these three parameters correctly classified $86.2 \%$ of ovarian mucinous tumours, including $91.5 \%$ of POMTs and $72.2 \%$ of eMOMCs, with the highest Youden Index (63.7\%).

In conclusion, our study has verified that PAX8 (mAb) is a highly specific marker and, when combined with tumour size and laterality, can significantly improve the accuracy of discriminating POMTs from eMOMCs. Although several other candidate molecular markers are available for this differential diagnosis, few are favourably specific and commercially available, thus greatly limiting their application. ${ }^{9}$ We propose a simple, convenient and high performance to price ratio algorithm combining PAX8 (mAb) immunostaining with tumour size and laterality. Although it should be validated in further studies, our algorithm may have a role in improving the diagnostic criteria for distinguishing between primary and metastatic mucinous ovarian carcinomas.

\section{Take home messages}

- Anti-PAX8 rabbit monoclonal antibody (mAb) demonstrated superior specificity than anti-PAX8 rabbit polyclonal antibody by immunohistochemistry.

- PAX8 (mAb), laterality and tumour size were useful in differentiating primary from extragenital metastatic mucinous ovarian tumours.

- Combining PAX8 (mAb) immunostaining status with tumour size and laterality is strongly recommended as an effective algorithm in routine practice.
Handling editor Runjan Chetty

Contributors $A H, H L$ and $L Z$ performed the experiments. $A H, H L$ and $Y L$ collected the clinical samples. $\mathrm{AH}, \mathrm{CR}$ and $\mathrm{YW}$ analysed the data. $\mathrm{AH}$ and $\mathrm{CL}$ wrote the paper. $\mathrm{CL}$ designed the project.

Funding This study was supported by the National Natural Science Foundation of China (grant No 81472430) and the National Key Scientific Instruments and Equipment Development Program of China (2013YQ03065108).

\section{Competing interests None.}

Ethics approval The study was approved by Peking University Health Science Centre.

Provenance and peer review Not commissioned; externally peer reviewed.

Open Access This is an Open Access article distributed in accordance with the Creative Commons Attribution Non Commercial (CC BY-NC 4.0) license, which permits others to distribute, remix, adapt, build upon this work non-commercially, and license their derivative works on different terms, provided the original work is properly cited and the use is non-commercial. See: http://creativecommons.org/ licenses/by-nc/4.0/

\section{REFERENCES}

1 Leen SL, Singh N. Pathology of primary and metastatic mucinous ovarian neoplasms. J Clin Pathol 2012;65:591-5.

2 Lee $\mathrm{KR}$, Young $\mathrm{RH}$. The distinction between primary and metastatic mucinous carcinomas of the ovary: gross and histologic findings in 50 cases. Am J Surg Pathol 2003:27:281-92.

3 Seidman JD, Kurman RJ, Ronnett BM. Primary and metastatic mucinous adenocarcinomas in the ovaries: incidence in routine practice with a new approach to improve intraoperative diagnosis. Am J Surg Pathol 2003;27:985-93.

4 Ronnett BM, Kurman RJ, Shmookler BM, et al. The morphologic spectrum of ovarian metastases of appendiceal adenocarcinomas: a clinicopathologic and immunohistochemical analysis of tumors often misinterpreted as primary ovarian tumors or metastatic tumors from other gastrointestinal sites. Am J Surg Pathol 1997;21:1144-55.

5 Young $\mathrm{RH}$, Hart WR. Metastases from carcinomas of the pancreas simulating primary mucinous tumors of the ovary. A report of seven cases. Am I Surg Pathol 1989:13:748-56.

6 Daya D, Nazerali L, Frank GL. Metastatic ovarian carcinoma of large intestinal origin simulating primary ovarian carcinoma. A clinicopathologic study of 25 cases. Am J Clin Pathol 1992:97:751-8.

7 Frumovitz M, Schmeler KM, Malpica A, et al. Unmasking the complexities of mucinous ovarian carcinoma. Gynecol Oncol 2010;117:491-6.

8 Yemelyanova AV, Vang $\mathrm{R}$, Judson $\mathrm{K}$, et al. Distinction of primary and metastatic mucinous tumors involving the ovary: analysis of size and laterality data by primary site with reevaluation of an algorithm for tumor classification. Am J Surg Pathol 2008;32:128-38.

9 McCluggage WG. Immunohistochemistry in the distinction between primary and metastatic ovarian mucinous neoplasms. J Clin Pathol 2012;65:596-600. 
10 Poleev A, Fickenscher $H$, Mundlos $S$, et al. PAX8, a human paired box gene: isolation and expression in developing thyroid, kidney and Wilms' tumors. Development 1992;116:611-23.

11 Maulbecker CC, Gruss P. The oncogenic potential of Pax genes. Embo J 1993;12:2361-7.

12 Bouchard M, Souabni A, Mandler M, et al. Nephric lineage specification by Pax2 and Pax8. Genes Dev 2002;16:2958-70.

13 Ozcan A, Shen SS, Hamilton C, et al. PAX 8 expression in non-neoplastic tissues, primary tumors, and metastatic tumors: a comprehensive immunohistochemical study. Mod Pathol 2011;24:751-64.

14 Nonaka D, Chiriboga L, Soslow RA. Expression of pax8 as a useful marker in distinguishing ovarian carcinomas from mammary carcinomas. Am J Surg Pathol 2008:32:1566-71.

15 Laury AR, Hornick JL, Perets $R$, et al. PAX8 reliably distinguishes ovarian serous tumors from malignant mesothelioma. Am J Surg Pathol 2010;34:627-35.

16 Ozcan A, Liles N, Coffey D, et al. PAX2 and PAX8 expression in primary and metastatic Mullerian epithelial tumors: a comprehensive comparison. Am J Surg Pathol 2011;35:1837-47.

17 Laury AR, Perets $\mathrm{R}$, Piao $\mathrm{H}$, et al. A comprehensive analysis of PAX8 expression in human epithelial tumors. Am J Surg Pathol 2011;35:816-26.

18 Tacha D, Zhou D, Cheng L. Expression of PAX8 in normal and neoplastic tissues: a comprehensive immunohistochemical study. Appl Immunohistochem Mol Morphol 2011;19:293-9.

19 Bowen NJ, Logani S, Dickerson EB, et al. Emerging roles for PAX8 in ovarian cancer and endosalpingeal development. Gynecol Oncol 2007;104:331-7.

20 Chu PG, Chung L, Weiss LM, et al. Determining the site of origin of mucinous adenocarcinoma: an immunohistochemical study of 175 cases. Am J Surg Pathol 2011:35:1830-6.

21 Tabrizi AD, Kalloger SE, Köbel M, et al. Primary ovarian mucinous carcinoma of intestinal type: significance of pattern of invasion and immunohistochemical expression profile in a series of 31 cases. Int J Gynecol Pathol 2010;29:99-107.
22 Köbel M, Kalloger SE, Boyd N, et al. Ovarian carcinoma subtypes are different diseases: implications for biomarker studies. PLoS Med 2008;5:e232.

23 Liliac L, Carcangiu ML, Canevari S, et al. The value of PAX8 and WT1 molecules in ovarian cancer diagnosis. Rom J Morphol Embryol 2013;54:17-27.

24 Moretti L, Medeiros LJ, Kunkalla K, et al. N-terminal PAX8 polyclonal antibody shows cross-reactivity with $\mathrm{N}$-terminal region of PAX5 and is responsible for reports of PAX8 positivity in malignant lymphomas. Mod Pathol 2012;25:231-6.

25 Lee KR, Scully RE. Mucinous tumors of the ovary: a clinicopathologic study of 196 borderline tumors (of intestinal type) and carcinomas, including an evaluation of 11 cases with 'pseudomyxoma peritonei'. Am J Surg Pathol 2000;24:1447-64.

26 Riopel MA, Ronnett BM, Kurman RJ. Evaluation of diagnostic criteria and behavior of ovarian intestinal-type mucinous tumors: atypical proliferative (borderline) tumors and intraepithelial, microinvasive, invasive, and metastatic carcinomas. Am J Surg Pathol 1999;23:617-35.

27 Park SH, Goo JM, Jo CH. Receiver operating characteristic (ROC) curve: practical review for radiologists. Korean J Radiol 2004;5:11-18.

28 Zaino RJ, Brady MF, Lele SM, et al. Advanced stage mucinous adenocarcinoma of the ovary is both rare and highly lethal: a Gynecologic Oncology Group study. Cancer 2011;117:554-62.

29 Tong GX, Devaraj K, Hamele-Bena D, et al. Pax8: a marker for carcinoma of Mullerian origin in serous effusions. Diagn Cytopathol 2011;39:567-74.

30 Levanon $\mathrm{K}, \mathrm{Ng} \mathrm{V}$, Piao HY, et al. Primary ex vivo cultures of human fallopian tube epithelium as a model for serous ovarian carcinogenesis. Oncogene 2010;29:1103-13.

31 Shan W, Mercado-Uribe I, Zhang J, et al. Mucinous adenocarcinoma developed from human fallopian tube epithelial cells through defined genetic modifications. Cell Cycle 2012;11:2107-13.

32 Seidman JD, Yemelyanova A, Zaino RJ, et al. The fallopian tube-peritoneal junction: a potential site of carcinogenesis. Int J Gynecol Pathol 2011;30:4-11. 